\title{
Local Interference Pricing for Distributed Beamforming in MIMO Networks
}

\author{
Changxin Shi, Randall A. Berry, and Michael L. Honig \\ Department of Electrical Engineering and Computer Science \\ Northwestern University, Evanston, Illinois 60208 \\ Email: cshi@u.northwestern.edu, \{rberry, mh\}@eecs.northwestern.edu
}

\begin{abstract}
We study a distributed algorithm for adjusting beamforming vectors in a peer-to-peer wireless network with multiple-input multiple-output (MIMO) channels. Each transmitter precoding matrix has rank one, and a linear minimum mean squared error (MMSE) filter is applied at each receiver. Our objective is to maximize the total utility summed over all users, where each user's utility is a function of the received signal-to-interference-plus-noise ratio (SINR). Given all users' beamforming vectors and receive filters, each receiver announces an interference price, representing the marginal cost of interference from other users. A particular transmitter updates its beamforming vector to maximize its utility minus the interference cost to other users. We show that if the utility functions satisfy certain concavity conditions, then the total utility is non-decreasing with each update. We also present numerical results that illustrate the effect of ignoring interference prices from all but the closest users, and relaxing requirements on the frequency of beam and price updates.
\end{abstract}

\section{INTRODUCTION}

Achieving high spectral efficiencies in multiuser wireless networks (e.g., cellular and wireless ad-hoc networks) depends critically on the application of interference mitigation techniques. When users in the network are equipped with multiple transmitters and receivers, the additional spatial degrees of freedom can be exploited to reduce interference. Joint optimization of power and spatial beams becomes especially challenging in peer-to-peer networks without centralized resource management, since an optimal allocation of resources at a particular transmitter (e.g., that maximizes total rate) generally requires information about other nodes.

We consider a peer-to-peer network with multiple-input multiple-output (MIMO) links. The performance of each transmitter-receiver pair is measured by a utility function,

This work was supported by ARO under grant W911NF-06-1-0339 and DARPA under grant W911NF-07-1-0028. which is a function of the transmission rate and depends on the transmitter's precoding matrix and the received interference-plus-noise covariance matrix. Our objective is to maximize the total (sum) utility over all users by applying a distributed algorithm to adjust the users' precoding matrices. We assume that each user's precoding matrix has rank one, i.e., the precoding matrix is a beamforming vector. This case has practical significance, and simplifies the optimization problem.

The algorithm we study is motivated by the work in [1]-[3]. In [1], the asynchronous distributed pricing (ADP) algorithm was presented for distributed power allocation in single-antenna wireless networks. In that algorithm, each user announces an interference price to all other users, which is the user's marginal change in utility per unit interference power. Given the interference prices from all interfering users, each user updates his power and beam by optimizing his utility minus the interference cost to other users, which is determined from their announced interference prices. It is shown in [1] that the ADP algorithm converges for a suitable class of utility functions. The proof is based on relating the updates in the distributed algorithm to best response updates in a supermodular game.

The ADP algorithm allows completely asynchronous updates of each users transmission power and prices. In [2] it is shown that with further restrictions on the updates, the ADP algorithm will converge monotonically for a broader class of utility functions. Furthermore, the analysis in [2] is shown to apply to a generalization of the ADP algorithm for multiple-input-single-output (MISO) networks. Similar distributed algorithms are studied for MIMO networks in [3], which have no restriction on the rank of the precoding matrix. These algorithms are observed to perform well numerically, although finding analytical conditions for convergence remains an open problem. The algorithm we consider here is similar to this, except we restrict the rank of the precoding matrix to be one. 
As in [1]-[3] we assume that interference is treated as noise. The goal is to optimize the total utility by selecting an optimal beamforming vector for each transmitter, assuming a linear minimum mean squared error (MMSE) receive filter for each associated receiver. Unlike the MISO network considered in [2], the receiver MMSE filters must be iteratively updated along with beams. Based on the convergence analysis in [2], it is straightforward to establish convergence with an appropriate update rule. We also numerically study the effect of limiting the information exchange. Specifically, we consider the scenarios in which users update their beamformers, ignoring interference prices and cross channel information from all but the closest (local) users. We also study the performance with infrequent price and receiver updates.

In terms of related work, the performance of the ADP algorithm and the effect of limiting the amount of information exchange for single-antenna links are studied through simulations in [4]. The Pareto-optimal rate-pair for a twouser MISO interference channel is characterized in [5]. For resource allocation techniques in MIMO networks, iterative waterfilling has been extensively studied (e.g., [6]-[8]), in which nodes implement best response updates without exchanging channel or interference information. However, iterative waterfilling is suboptimal in terms of the overall network performance.

In the next section, we present the system model and resource optimization problem. In Section III, we propose a distributed algorithm and consider some variations with reduced information exchange. Simulation results are presented in Section IV, and conclusions are given in Section V.

\section{System MODEL}

We consider a time-invariant wireless network with $K$ pairs of transmitters and receivers, where each transmitter has $N_{T}$ antennas and each receiver has $N_{R}$ antennas. Given channel matrices $\mathbf{H}_{i k}$ 's, representing the channel from transmitter $k$ to receiver $i$, the received signal at receiver $k$ is given by

$$
\mathbf{y}_{k}=\mathbf{H}_{k k} \mathbf{x}_{k}+\sum_{i \neq k} \mathbf{H}_{k i} \mathbf{x}_{i}+\mathbf{n}_{k}
$$

where $\mathbf{x}_{k}$ is the transmit signal vector for user $k$ and $\mathbf{n}_{k}$ is additive complex Gaussian noise with covariance matrix $\mathbf{R}_{n_{k}}$. Assuming the interference is treated as additive Gaussian noise, the achievable rate for user $k$ is given by [9],

$$
R_{k}=\log \operatorname{det}\left(\mathbf{I}+\mathbf{H}_{k k}^{\mathrm{H}}\left(\mathbf{R}_{n_{k}}+\sum_{i \neq k} \mathbf{H}_{k i} \mathbf{Q}_{i} \mathbf{H}_{k i}^{\mathrm{H}}\right)^{-1} \mathbf{H}_{k k} \mathbf{Q}_{k}\right)
$$

where $\mathbf{Q}_{k}=\mathrm{E}\left[\mathbf{x}_{k} \mathbf{x}_{k}^{\mathrm{H}}\right]$ is the transmit covariance matrix for user $k$ and $(\cdot)^{\mathrm{H}}$ denotes Hermitian transpose. Generally, $\mathbf{Q}_{k}$ is determined by the precoding matrix $\mathbf{V}_{k}$ at the transmitter $k$. In this paper, we assume that each precoding matrix $\mathbf{V}_{k}$ is rank one, i.e., $\mathbf{V}_{k}=\mathbf{v}_{k}$ is a vector and $\mathbf{x}_{k}=\mathbf{v}_{k} x_{k}$ where $x_{k}$ is a single transmitted symbol with $\mathrm{E}\left[\left|x_{k}\right|^{2}\right]=1$.

With an optimized beamforming vector, the capacity in (2) can be achieved with a linear MMSE filter, given by

$$
\mathbf{g}_{k}=\left(\mathbf{R}_{n_{k}}+\sum_{i \neq k} \mathbf{H}_{k i} \mathbf{v}_{i} \mathbf{v}_{i}^{\mathrm{H}} \mathbf{H}_{k i}^{\mathrm{H}}\right)^{-1} \mathbf{H}_{k k} \mathbf{v}_{k} .
$$

The filtered signal is then

$$
\hat{y}_{k}=\mathbf{g}_{k}^{\mathrm{H}}\left(\mathbf{H}_{k k} \mathbf{v}_{k} x_{k}+\sum_{i \neq k} \mathbf{H}_{k i} \mathbf{v}_{i} x_{i}+\mathbf{n}_{i}\right),
$$

and the corresponding signal-to-interference-plus-noise ratio (SINR) for user $k$ can be written as

$$
\gamma_{k}=\left(\mathbf{H}_{k k} \mathbf{v}_{k}\right)^{\mathrm{H}}\left(\mathbf{R}_{n_{k}}+\sum_{i \neq k} \mathbf{H}_{k i} \mathbf{v}_{i} \mathbf{v}_{i}^{\mathrm{H}} \mathbf{H}_{k i}^{\mathrm{H}}\right)^{-1} \mathbf{H}_{k k} \mathbf{v}_{k} .
$$

The quality of service for each user is measured by a utility function of the transmission rate. Equivalently, we define the utility $u_{k}\left(\gamma_{k}\right)$ as a function of $\gamma_{k}$. We assume that for all $k$, the utility function is monotonically increasing, concave and twice differentiable function of $\gamma_{k}$. Our objective is to choose the beamforming vector $\mathbf{u}_{k}$ for each user $k$ to maximize the utility summed over all users, i.e.,

$$
\begin{aligned}
\max _{\mathbf{v}_{1}, \cdots, \mathbf{v}_{K}} & \sum_{k=1}^{K} u_{k}\left(\gamma_{k}\right) \\
\text { s.t. } & \left\|\mathbf{v}_{k}\right\|^{2} \leq P_{k}^{\text {max }}, \quad k=1, \ldots, K,
\end{aligned}
$$

where $\gamma_{k}$ is determined by the beamforming vectors via (5), and $P_{k}^{\max }$ denotes the power constraint for user $k$.

The nonlinear optimization problem $P$ is difficult to solve in general. Furthermore, we seek a distributed algorithm for solving this in which users do not know the entire network topology and the other users' utility functions. The users must then exchange limited information so that each user can locally decide on their own beamforming vector. As discussed in the introduction, our approach to this problem is based on a variation of the interference pricing algorithms studied in [2], [3]. In these algorithms, the limited information exchanged by the users is the interference prices, which reflect the marginal change in utility per unit interference power. A particular user then updates his beamforming vector to maximize his utility minus the interference cost to other users, which is determined from their announced interference prices. The exact distributed pricing algorithm we study here we refer to as the MIMO Distributed pricing algorithm (MIMO-DP). This details of the MIMO-DP are specified in the next section. 


\section{Distributed Pricing Algorithm}

\section{A. MIMO Distributed Pricing (MIMO-DP) Algorithm}

From (5), it is not clear what is the "interference power", and how a change in interference will influence the corresponding SINR and utility. However, if we fix each receive filter, the MIMO network reduces to the MISO case in which the channel vector from transmitter $k$ to receiver $i$ is $\mathbf{h}_{i k}^{\mathrm{H}}=\mathbf{g}_{i}^{\mathrm{H}} \mathbf{H}_{i k}$. Then, given a set of receive filters $\left\{\mathbf{g}_{1}, \mathbf{g}_{2}, \cdots, \mathbf{g}_{K}\right\}$, we can re-cast the algorithm in [2] to solve Problem $P$ with

$$
\gamma_{k}=\frac{\left|\mathbf{g}_{k}^{\mathrm{H}} \mathbf{H}_{k k} \mathbf{v}_{k}\right|^{2}}{\left|\mathbf{g}_{k}^{\mathrm{H}} \mathbf{n}_{k}\right|^{2}+\sum_{i \neq k}\left|\mathbf{g}_{k}^{\mathrm{H}} \mathbf{H}_{k i}^{\mathrm{H}} \mathbf{v}_{i}\right|^{2}} .
$$

Define the interference price for user $k$, given a fixed $\mathbf{g}_{k}$, as

$$
\pi_{k}=-\frac{\partial u_{k}\left(\gamma_{k}\right)}{\partial I_{k}}
$$

where $I_{k}=\sum_{i \neq k}\left|\mathbf{g}_{k}^{\mathrm{H}} \mathbf{H}_{k i} \mathbf{v}_{i}\right|^{2}$ is the interference power after the receive filter. Given fixed interference prices and the receive filters and beamforming vectors for the other users, transmitter $k$ then updates its beamforming vector by solving the subproblem:

$$
\begin{aligned}
\max _{\mathbf{v}_{k}} & u_{k}\left(\gamma_{k}\left(\mathbf{v}_{k} ; \mathbf{v}_{-k}\right)\right)-\sum_{i \neq k} \pi_{i}\left|\mathbf{g}_{i}^{\mathrm{H}} \mathbf{H}_{i k} \mathbf{v}_{k}\right|^{2} \\
& \text { s.t. }\left\|\mathbf{v}_{k}\right\|^{2} \leq P_{k}^{\max },
\end{aligned}
$$

where $\mathbf{v}_{-k}$ denotes the beamforming vectors of users other than user $k$.

If the users repeatedly update their beamforming vectors and interference prices according to $\mathrm{P}_{k}$ and 7, as in [2], then the MISO-DP algorithm will converge. Hence this gives (locally) optimal beamforming vectors, if we start with the optimal receive filters. However, the receiver updates must now be included in the analysis.

We formally state the MIMO-DP algorithm as follows:

1) Initialization: Each user (link) $k$ chooses an initial beamforming vector $\mathbf{v}_{k}$ satisfying the power constraint, and applies the corresponding MMSE filter (3) at the receiver.

2) Price Update: Each receiver $k$ calculates the interference price $\pi_{k}$ from (7) given the current beamforming vectors $\mathbf{v}_{k}$ and receive filters $\mathbf{g}_{k}$, and announces this price to every other user.

3) Beamformer Update: A randomly chosen user $k$ solves Problem $\mathrm{P}_{k}$ and updates his beamforming vector, given the interference prices $\left\{\pi_{i}\right\}_{i \neq k}$.

4) Receive Filter Update: Any set of users update their receive filters using (3), given beamforming vectors $\mathbf{v}_{k}$ and announce to other users.

5) Repeat from step 2).
In general, there may be multiple solutions to Problem $\mathrm{P}_{k}$, which gives the beamformer update. In that case, one of the solutions can be randomly chosen assuming the previous beam is not a solution. Otherwise, the previous beam is kept. The MIMO-DP algorithm assumes that each user $k$ knows the product $\mathbf{H}_{i k}^{\mathrm{H}} \mathbf{g}_{i}$ for all $i$, in addition to local information, namely, his own utility function and the interference-plus-noise power at the output of $\mathbf{g}_{k}$. Hence, receiver $k$ must announce $\mathbf{g}_{k}$ after each update. Later we show results illustrating the performance if each transmitter uses the initial values of $\mathbf{g}_{k}$ 's in $\mathrm{P}_{k}$, with no successive update or infrequent updates. Note that each transmitter does not need to know the other users' beamforming vectors or the channel matrices $\mathbf{H}_{i j}$ for $j \neq k$ and all $i$.

\section{B. Convergence}

The beamformer update in the MIMO-DP algorithm is the same as that in the algorithm in [3]. However, the algorithm in [3] updates both beams and associated powers, whereas here we assume the precoding matrices are rank one. As in [2], it can be shown that if the utility function satisfies that $-\frac{u^{\prime \prime}(\gamma) \gamma}{u^{\prime}(\gamma)} \in[0,2]$, then it is convex with respect to the received interference power. If all interference prices are current (i.e., have been updated since the last beamformer update), it can then be shown that a subsequent beamformer update cannot decrease the total utility. Also, updating the receive filter $\mathbf{g}_{k}$ further increases user $k$ 's SINR and utility without changing others' utilities. Hence the total utility must monotonically converge to a limit with beam and receiver updates. This is summarized in the following proposition

Proposition 1: If for each user $k,-\frac{u_{k}^{\prime \prime}\left(\gamma_{k}\right) \gamma_{k}}{u_{k}^{\prime}\left(\gamma_{k}\right)} \in[0,2]$ for all feasible $\gamma_{k}$, then the MIMO-DP algorithm converges to a stationary point, which satisfies the Karush-Kuhn-Tucker (KKT) conditions of Problem $P$ with MMSE receive filters.

\section{Solving $\mathrm{P}_{k}$}

In the MIMO-DP algorithm, each user must solve the subproblem $\mathrm{P}_{k}$ repeatedly, which is a nonlinear optimization problem. Since this may be time-consuming for large networks, we present an efficient numerical algorithm for solving that problem.

The KKT conditions for Problem $\mathrm{P}_{k}$ for user $k$ are

$$
\underbrace{\left[a_{k}\left(\mathbf{v}_{k}\right) \mathbf{H}_{k k}^{\mathrm{H}} \mathbf{g}_{k} \mathbf{g}_{k}^{\mathrm{H}} \mathbf{H}_{k k}-\sum_{i \neq k} \pi_{i} \mathbf{H}_{i k}^{\mathrm{H}} \mathbf{g}_{i} \mathbf{g}_{i}^{\mathrm{H}} \mathbf{H}_{i k}\right]}_{\mathbf{X}_{k}} \mathbf{v}_{k}=\lambda_{k} \mathbf{v}_{k},
$$

where

$$
a_{k}\left(\mathbf{v}_{k}\right)=\frac{u_{k}^{\prime}\left(\gamma_{k}\right)}{\left|\mathbf{g}_{k}^{\mathrm{H}} \mathbf{n}_{k}\right|^{2}+\sum_{i \neq k}\left|\mathbf{g}_{k}^{\mathrm{H}} \mathbf{H}_{k i} \mathbf{v}_{i}\right|^{2}}
$$


and $\lambda_{k}$ is the Lagrange multiplier associated with the power constraint. In general, $a_{k}$ is a function of $\mathbf{v}_{k}$. However, if the utility function is linear in the SINR, then the optimal beamforming vector $\mathbf{v}_{k}^{*}$ is either the eigenvector of the matrix $\mathbf{X}_{k}$ in (8) corresponding to its largest positive eigenvalue with an appropriate scale factor if the largest eigenvalue is positive, or is the zero vector if all eigenvalues are negative.

With this observation, we start with any feasible beamforming vector $\mathbf{v}_{k}^{0}$, and treat the coefficient $a_{k}$ as a constant. We then compute $\mathbf{v}_{k}^{1}$ by recomputing $a_{k}$ and finding the appropriate eigenvector of $\mathbf{X}_{k}$. Iterating we get the sequence $\left\{\mathbf{v}_{k}^{0}, \mathbf{v}_{k}^{1}, \cdots, \mathbf{v}_{k}^{n}, \cdots\right\}$. If it converges, then the limit point satisfies the KKT conditions. Although convergence is not guaranteed, the algorithm works well in our simulations.

\section{Localized Distributed Pricing Algorithm}

The MIMO-DP algorithm provides an efficient approach to optimize network performance. However, each user requires knowledge of cross channels and interference prices, which might incur large overhead. In addition, this overhead may provide little benefit for relatively weak (far away) users. On the other hand, to guarantee the convergence, we require users to follow the update rules. In what follows, we study the following modifications to the MIMO-DP algorithm.

1) Limited Information Exchange: In practice, we can modify the MIMO-DP algorithm by taking into account the interference cost to a limited number of interfering users in Problem $\mathrm{P}_{k}$ (those that are relatively close). The criterion for making this reduction depends on the cost of exchanging prices. This corresponds to limited power for price updates. Obviously, keeping all channel and price information gives the best performance. In Section IV, we examine the performance in different scenarios.

2) Localized Update Rule: The convergence proof for the MIMO-DP algorithm relies on the fact that whenever one user updates his beamformer, he knows the current interference prices from every user in the network. To reduce the overhead required to broadcast these prices, it is desirable to relax this restriction and allow multiple users to simultaneously update their beamformers before new prices are announced. In [2] it is observed that such simultaneous updates can cause oscillations and prevent convergence with another choice for the utility function. Here, in an attempt to avoid this, we only allow users to update simultaneously provided that they are sufficiently far away from each other. In other words, within a threshold distance from any updating user, there can be no other user updating his beam. (In practice, the undesired simultaneous update can be avoided by sensing a beacon signal, which indicates that a user in the vicinity is updating.) With this restriction, although there is no proof to guarantee convergence, the MIMO-DP algorithm still converges in all cases we simulated with the rate utility function.

\section{Simulation Results}

In this section, we first show a typical convergence plot for the MIMO-DP algorithm in Section III-A. Then the performance of algorithms with the variations presented in Section III-D are shown and discussed.

\section{A. MIMO Distributed Pricing Algorithm}

We consider a MIMO network of 5 users with 3 transmit and 3 receive antennas for each user, randomly placed in a square of $100 \mathrm{~m} \times 100 \mathrm{~m}$. The direct and cross channel matrices have i.i.d. complex Gaussian entries with each variance determined by the attenuation due to path-loss. Namely, the average gain (received power) of each single link is $\sigma^{2}(d)=\sigma_{0}^{2}\left(\frac{d}{d_{0}}\right)^{-4}$, where $d_{0}=10 \mathrm{~m}$ is the reference distance and also the minimum distance allowed between any transmitter and receiver, and $\sigma_{0}^{2}$ is the reference power. The maximum power, noise, and $\sigma_{0}$ are selected so that the expected received signal-to-noise ratio (SNR) of a single link with a separation of $10 \mathrm{~m}$ is $100(20 \mathrm{~dB})$. The rate utility function, $u_{k}\left(\gamma_{k}\right)=\log \left(1+\gamma_{k}\right)$ is assumed for all users.

Figure 1 shows the sum rate versus the number of iterations for a particular model realization achieved by applying the MIMO-DP algorithm. We also examine the scenario in which receive filter updates are only announced every $n$ iterations (even though the filters are updated after every beam update). In other words, the transmitter uses obsolete information for the receive filters when updating its beamformers. The algorithm starts from an arbitrary selection of beamformers, and converges to a stationary point, predicted by Proposition 1. To check whether the limit point is indeed optimal, we use MATLAB to solve the global optimization problem $P$ directly, starting from the limit point achieved by the MIMO-DP algorithm. The total utility obtained in this way is indicated by the dash-dot line in Fig. 1, which matches the limit point of the MIMODP algorithm. The figure shows that the more frequently the receive filter updates are announced, the faster the algorithm converges.

\section{B. Effects of Limited Information Exchange}

Here, we consider the scenario where users ignore prices announced by remote users.

Similar to the previous subsection, now we consider a MIMO network of $N$ users with 3 transmitter antennas and 3 receive antennas for each user, in which each transmitter and the associated receiver are randomly placed at nodes 


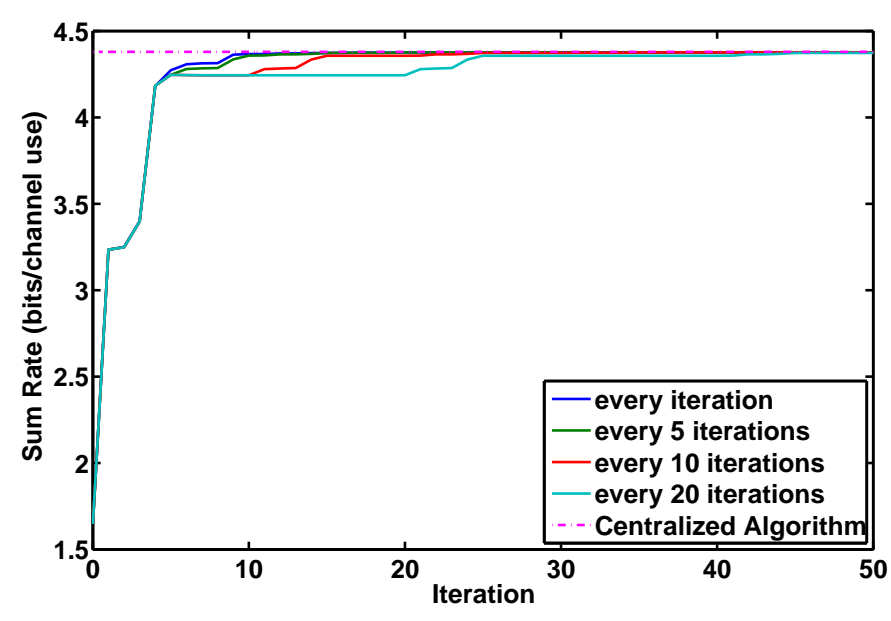

Fig. 1. Illustration of the convergence of the MIMO-DP algorithm with different periods for announcing receive filter updates.

in a grid of $200 \mathrm{~m} \times 200 \mathrm{~m}$ formed from minimum squares of $10 \mathrm{~m} \times 10 \mathrm{~m}$ according to a uniform distribution. Channel matrices are configured the same way as before with the reference SNR at $10 \mathrm{~m}$ set to be $30 \mathrm{~dB}$.

We still assume each user $k$ knows the channel matrices $\mathbf{H}_{i k}$ for all $i$. However, when each user updates his beamformer by solving Problem $\mathrm{P}_{k}$, he ignores the interference cost to user $i$ if $y_{i k}=\mathbf{1}^{\mathrm{H}} \mathbf{H}_{i k}^{\mathrm{H}} \mathbf{H}_{i k} \mathbf{1}$ is smaller than some threshold, where $\mathbf{1}$ is an all-one vector. The reason to choose this quantity as the criterion is as follows. Consider the feedback link from receiver $i$ to transmitter $k$ for broadcasting price $\pi_{i}$. If we assume the channel is reciprocal and receiver $i$ uses the all-one vector as its beamforming vector, from (5), the received SINR at transmitter $k$ is determined by $y_{i k}$, assuming the interference and noise covariance matrix $\mathbf{R}_{n}$ is a scaled identity matrix. Hence, this quantity should be sufficiently large to enable reliable reception of $\pi_{i}$.

Figs. 2 and 3 show the average rate per user versus number of users (equivalently density) with different thresholds, averaged over 100 channel realizations. In Fig. 2, all receivers are uniformly distributed (as are the transmitters), whereas for Fig. 3 each associated receiver is randomly placed within a $100 \mathrm{~m} \times 100 \mathrm{~m}$ square centered around its transmitter. To interpret these results in terms of local interference, we convert the threshold with respect to channel gain into a threshold with respect to distance. Specifically, the distance threshold means a single-antenna link with this separation without considering Rayleigh fading will have the same received SNR as the MIMO link using the original threshold for $y_{i k}$. In these figures, "threshold $=\infty$ " corresponds to the original MIMO-DP algorithm in which all interfering users' prices are included, while "threshold $=0 \mathrm{~m}$ " corresponds to an algorithm with-

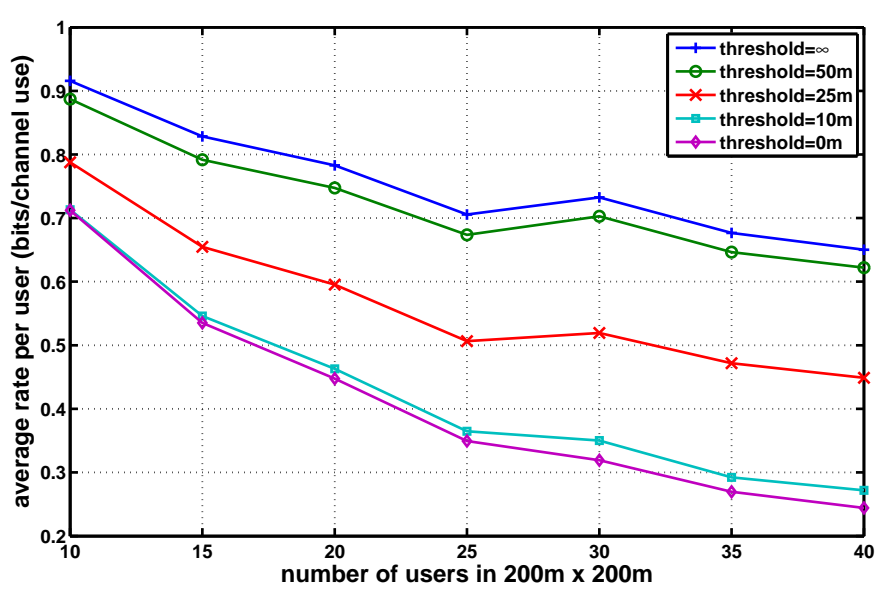

Fig. 2. Illustration of the effect of ignoring interference prices from remote users (receivers are uniformly distributed).

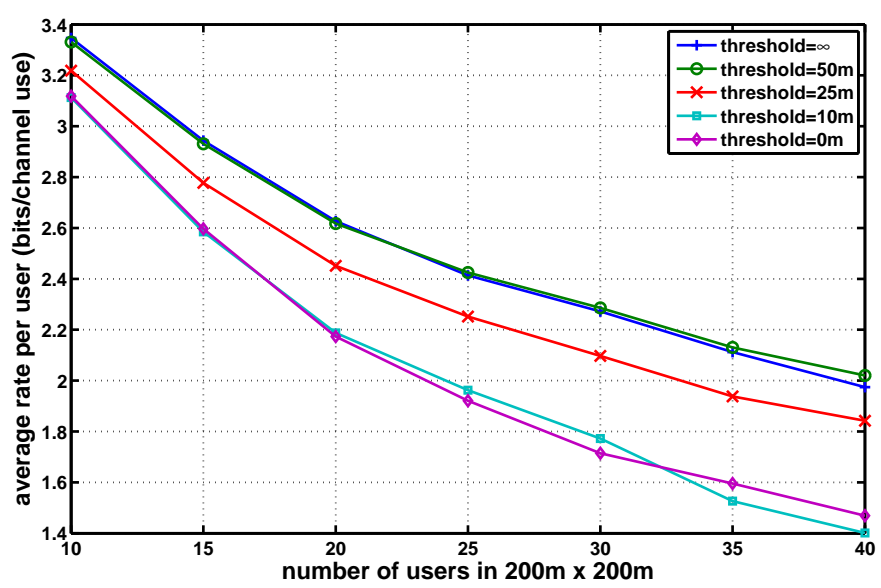

Fig. 3. Illustration of the effect of ignoring interference prices from remote users (each receiver is near its transmitter).

out information exchange: each user maximizing his own utility without considering any interference cost. The figures show that a reasonably small threshold $(50 \mathrm{~m})$ does not hurt the performance significantly. Furthermore, although the average rate per user is higher when each receiver is located near its transmitter (Fig. 3), the benefit of exchanging price compared to the myopic strategy without information exchange is more significant when that receivers are uniformly distributed (Fig. 2). This is because when each receiver is located near its transmitter, the rate reduction due to interference is smaller.

\section{Localized MIMO-DP Algorithm}

We now show results assuming simultaneous beamformer updates can occur outside a given radius from any updating transmitter. When the distance between two updating transmitters $j$ and $k$ is large, the change of the interference price of a particular interfering user due to transmitter $j$ 's update is negligible for the other transmitter $k$ and vice 


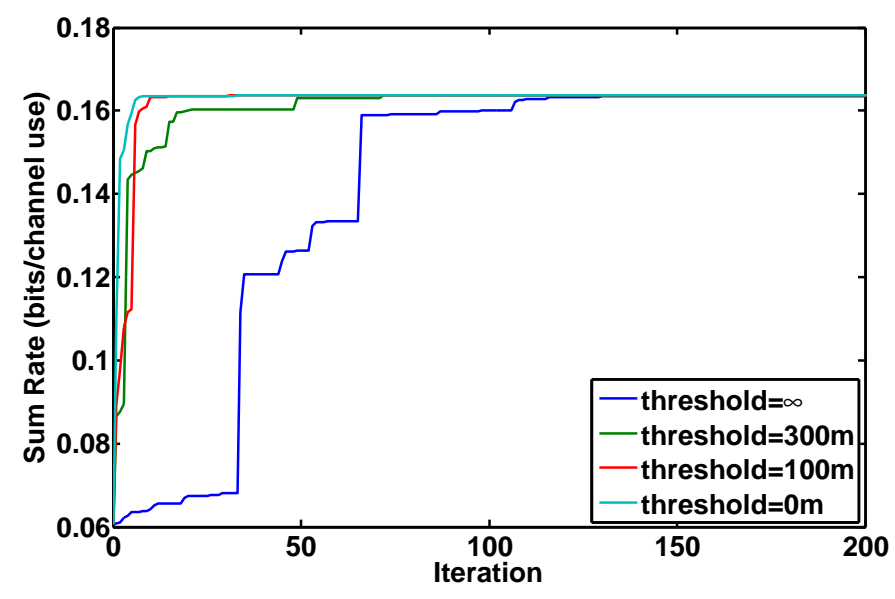

Fig. 4. Illustration of the effect of allowing faraway users to update beamformers simultaneously.

versa, because that interfering user is at least far away from one of the transmitters $j$ and $k$. Hence multiple users can update at the same time as long as they are far enough away. We simulated a network of 20 users within a square of $500 \mathrm{~m}$ $\mathrm{x} 500 \mathrm{~m}$ with the same assumptions as in Section IV-A. In all cases simulated, no matter how small the threshold is, we observed convergence of the distributed algorithm. Fig. 4 shows typical convergence performance with different distance thresholds (although the convergence has not been proven analytically). "Threshold $=\infty$ " corresponds to the original MIMO-DP algorithm, while "threshold $=0 \mathrm{~m}$ " refers to the scenario where simultaneous beamformer updates are completely unrestricted. Furthermore, according to these numerical results, the speed of convergence is faster when simultaneous updates are allowed.

\section{Conclusions}

We have presented a distributed algorithm for adjusting beamforming vectors in a peer-to-peer MIMO network to maximize the sum utility over all users, with rank-one precoding matrices. Convergence is established based on the result in [2] that each beamformer update increases the total utility, provided that all interference prices are current, and the observation that updating receive filters increases those users' utilities while others' utilities are unchanged. Examples were also presented, which indicate that ignoring some prices related to remote users, or relaxing the update rules does not compromise the performance of the MIMODP algorithm significantly.

Due to the spatial degrees of freedom in MIMO networks, it is not optimal in general to apply a rank-one precoding matrix for each user. Therefore, how to adjust a precoding matrix to approach an optimal solution in a MIMO network without rank estimation is still open. In addition, the convergence rate as a function of system parameters has not been considered.

\section{REFERENCES}

[1] J. Huang, R. A. Berry, and M. L. Honig, "Distributed interference compensation for wireless networks," IEEE Journal on Selected Areas in Communications, vol. 24, no. 5, pp. 1074-1084, May 2006.

[2] C. Shi, R. A. Berry, and M. L. Honig, "Monotonic Convergence of Distributed Interference Pricing in Wireless Networks," in Proc. IEEE International Symposium on Information Theory, June 2009.

[3] C. Shi, D. A. Schmidt, R. A. Berry, M. L. Honig, and W. Utschick, "Distributed Interference Pricing for the MIMO Interference Channel," in Proc. IEEE International Conference on Communications, June 2009.

[4] J. Huang, R. A. Berry, and M. L. Honig, "Performance of Distributed Utility-Based Power Control for Wireless Ad Hoc Networks," in Proc. Military Communications Conference, 2005, pp. 2481-2487, Oct 2005 .

[5] E. Larsson and E. Jorswieck, "Competition versus cooperation on the MISO interference channel," IEEE Journal on Selected Areas in Communications, vol. 26, no. 7, pp. 1059-1069, Sept. 2008

[6] M. F. Demirkol and M. A. Ingram, "Power-controlled capacity for interfering MIMO links," in Proc. IEEE Vehicular Technology Conference, vol. 1, Oct. 2001.

[7] S. Ye and R. S. Blum, "Optimized signaling for MIMO interference systems with feedback," IEEE Trans. Signal Processing, vol. 51, no. 11, pp. 2839-2848, Nov. 2003.

[8] G. Scutari, D. P. Palomar and S. Barbarossa, "Competitive design of multiuser MIMO systems based on game theory: A unified view," IEEE Journal on Selected Areas in Communications: Special Issue on Game Theory, vol. 26, no. 7, pp. 1089-1103, Sept. 2008.

[9] E. Telatar, "Capacity of Multi-Antenna Gaussian Channels," Eur. Trans. Telecomm. ETT, vol. 10, no. 6, pp. 585-596, Nov. 1999. 